\title{
Associations Between Serum Vitamin D and Adverse Pathology in Men Undergoing Radical Prostatectomy
}

Yaw A. Nyame, Adam B. Murphy, Diana K. Bowen, Gregory Jordan, Ken Batai, Michael Dixon, Courtney M.P. Hollowell, Stephanie Kielb, Joshua J. Meeks, Peter H. Gann, Virgilia Macias, Andre Kajdacsy-Balla, William J. Catalona, and Rick Kittles

Yaw A. Nyame, Cleveland Clinic, Cleveland, Ohio; Adam B. Murphy, Diana K. Bowen, Gregory Jordan, Michael Dixon, Stephanie Kielb, Joshua J. Meeks, and William J. Catalona, Northwestern University, Chicago; Courtney M.P. Hollowell, Cook County Health and Hospitals System; Peter H. Gann, Virgilia Macias, and Andre Kajdacsy-Balla, University of Illinois at Chicago, Chicago, L; and Ken Batai and Rick Kittles, University of Arizona, Tucson, AZ. Published online ahead of print at www.jco.org on February 22, 2016

Supported by a grant from the US Department of Defense W81XWH-10-10532 pd22E (A.B.M.), and the following National Institutes of Health grants: 1R01MD007105-01 (R.K.); IK2CX000926-01 (A.B.M.), and P50 CA090386-10S1 (W.J.C.)

Y.A.N., A.B.M., and D.K.B. contributed equally to the drafting of this manuscript.

Authors' disclosures of potential conflicts of interest are found in the article online at www.jco.org. Author contributions are found at the end of this article.

Corresponding author: Adam B. Murphy, MD, Northwestern University, Tarry Building Room 16-703, 300 E Superior Chicago, IL 60611; e-mail: a-murphy2@ northwestern.edu.

(C) 2016 by American Society of Clinical Oncology

0732-183X/16/3412w-1345w/\$20.00

DOI: $10.1200 / J C O .2015 .65 .1463$

\section{$\begin{array}{llllllll}\text { A } & \text { B } & \mathbf{S} & \mathbf{T} & \mathbf{R} & \text { A } & \text { C } & \mathbf{T}\end{array}$}

\section{Purpose}

Lower serum vitamin $\mathrm{D}$ levels have been associated with an increased risk of aggressive prostate cancer. Among men with localized prostate cancer, especially with low- or intermediate-risk disease, vitamin D may serve as an important biomarker of disease aggression. The aim of this study was to assess the relationship between adverse pathology at the time of radical prostatectomy and serum 25-hydroxyvitamin D (25-OH D) levels.

\section{Methods}

This cross-sectional study was carried out from 2009 to 2014, nested within a large epidemiologic study of 1,760 healthy controls and men undergoing prostate cancer screening. In total, 190 men underwent radical prostatectomy in the cohort. Adverse pathology was defined as the presence of primary Gleason 4 or any Gleason 5 disease, or extraprostatic extension. Descriptive and multivariate analyses were performed to assess the relationship between $25-\mathrm{OH} D$ and adverse pathology at the time of prostatectomy.

\section{Results}

Eighty-seven men (45.8\%) in this cohort demonstrated adverse pathology at radical prostatectomy. The median age in the cohort was 64.0 years (interquartile range, 59.0 to 67.0). On univariate analysis, men with adverse pathology at radical prostatectomy demonstrated lower median serum 25-OH D (22.7 v $27.0 \mathrm{ng} / \mathrm{mL}, P=.007)$ compared with their counterparts. On multivariate analysis, controlling for age, serum prostate specific antigen, and abnormal digital rectal examination, serum $25-\mathrm{OH} D$ less than $30 \mathrm{ng} / \mathrm{mL}$ was associated with increased odds of adverse pathology (odds ratio, $2.64 ; 95 \% \mathrm{Cl}, 1.25$ to $5.59 ; P=.01$ ).

\section{Conclusion}

Insufficiency/deficiency of serum 25-OH D is associated with increased odds of adverse pathology in men with localized disease undergoing radical prostatectomy. Serum 25-OH D may serve as a useful biomarker in prostate cancer aggressiveness, which deserves continued study.

\section{J Clin Oncol 34:1345-1349. (C) 2016 by American Society of Clinical Oncology}

\section{INTRODUCTION}

Recent population-based studies suggest that there is a relationship between vitamin $\mathrm{D}$ deficiency and increased prostate cancer $(\mathrm{PCa})$ risk. ${ }^{1}$ In addition, there is literature that demonstrates that vitamin $\mathrm{D}$ deficiency is also associated with aggressive $\mathrm{PCa}{ }^{2,3}$ Interestingly, the prevalence of vitamin $\mathrm{D}$ deficiency in a cohort of men in Chicago, Illinois-a low UV exposure location-was $41.2 \%$, and the prevalence of deficiency was significantly higher among black men. ${ }^{4}$ The relationship between vitamin $\mathrm{D}$ and PCa may explain some disparities seen in $\mathrm{PCa}$, especially among black men.
The majority of men diagnosed with PCa in the United States present with PCa localized to the prostate gland. The current treatment paradigm is shifting to managing these men with a surveillance protocol. Active surveillance as a management strategy of low-risk PCa-defined by the criteria of Epstein et $\mathrm{al}^{5}$ and D'Amico et $\mathrm{al}^{6}$ - is an evolving strategy that relies on risk stratification and diagnostic testing. It is unclear whether there are high-risk populations, such as black men, who would benefit from additional screening tests before management with a surveillance protocol. However, useful clinical risk factors have been extrapolated from pathologic findings from radical prostatectomy specimens. ${ }^{7,8}$ For example, a review 
of the radical prostatectomy database at Johns Hopkins Hospital demonstrated that black men with very low-risk PCa were more likely to have adverse pathology at prostatectomy compared with men of European descent. ${ }^{9}$

There is limited literature on the relationship between vitamin $\mathrm{D}$ and pathologic findings at the time of radical prostatectomy. ${ }^{10}$ The aim of this study was to determine whether serum 25-hydroxyvitamin D (25-OH D) correlates with adverse pathology at radical prostatectomy among a diverse population of men from a large, urban population.

\section{METHODS}

This cross-sectional, observational study evaluating the associations of serum $25-\mathrm{OH} \mathrm{D}$ status with adverse pathology in men undergoing radical prostatectomy was carried out from 2009 to 2014. It was nested within a large epidemiologic study of 1,760 healthy controls and men undergoing PCa screening that evaluated environmental and biologic mediators of vitamin $\mathrm{D}$ and PCa risk. A total of 812 men between the ages of 40 and 79 years underwent prostate core biopsy for increasing prostate specific antigen (PSA) levels and/or an abnormal finding on digital rectal examination. All participants were prospectively enrolled through outpatient urology clinics from three academic (Northwestern University, University of Chicago, University of Illinois at Chicago) and two public (Jesse Brown VA Medical Center and Cook County Hospital) institutions in Chicago, Illinois.

One hundred ninety men were included in our study after undergoing radical prostatectomy for diagnosis of clinically localized PCa within 1 year of their positive prostate biopsy. Genitourinary pathologists reviewed all pathologic specimens. Men with a diagnosis of PCa were excluded from analysis if they received adjuvant therapy prior to radical prostatectomy or if they underwent treatment at a nonstudy institution. All study participants provided written consent, and the institutional review board at each participating institution approved the protocol.

\section{Clinical and Environmental Data}

Trained research coordinators collected all patient data via patient questionnaires and independent chart review. 25-OH D was collected by a peripheral serum sample at the time of enrollment by a trained research coordinator or clinic phlebotomists. Serum 25-OH D levels were measured using the DiaSorin Liaison 25-OH Vitamin D TOTAL Assay platform (DiaSorin, Stillwater, MN) by a direct, competitive chemiluminescent immunoassay. Relevant clinical covariates included age, first-degree family history of $\mathrm{PCa}$, and tobacco use. Indicators of socioeconomic status were collected through a combination of a questionnaire and medical record review. Information on ethnicity and race was collected by self-identification and was characterized as black, white, or other. In addition, body mass index (BMI) was calculated from the measurement of standing height (in meters) and weight (in kilograms) of all participants at enrollment.

Cancer-specific clinical data, including biopsy result (ie, Gleason score, number of positive cores, and percentage of core involvement), clinical stage (tumor, node, metastasis classification), and PSA were recorded, and men in the cohort were classified according to the National Comprehensive Cancer Network (NCCN) risk classification groups. ${ }^{11}$ Patients were then evaluated on the basis of the presence or absence of adverse pathologic features at the time of radical prostatectomy. Adverse pathology was defined by the presence of dominant Gleason pattern 4, the presence of any pattern 5 , and pathologic stage $\geq$ pT3aN0M0. ${ }^{12}$ All analyses were stratified by ethnicity/race and NCCN risk classification.

\section{Statistical Analysis}

Descriptive statistics were used to characterize important covariates, including age, serum 25-OH D level, serum PSA level, race, BMI, tobacco use, income, first-degree PCa family history, marital status, education, and 5 alpha-reductase inhibitor (5-ARI) use among men with and without adverse pathology at radical prostatectomy. 5-ARI-adjusted PSA values were calculated by doubling the prebiopsy PSA value. ${ }^{13}$ A sample size of 190 patients-assuming a 1:1 case-control ratio-in a population with a prevalence of adverse pathology of $35 \%$ had a power of $87.7 \%$ to detect an OR of 2.5 or greater.

Continuous covariates were compared using a Wilcoxon rank sum test, and categorical variables were compared using a $\chi^{2}$ test. Multivariate analysis was conducted using binary logistic regression to further evaluate the association of $25-\mathrm{OH} \mathrm{D}$ with adverse pathology. All multivariate analyses were adjusted for season of blood draw (ie, high $v$ low ultraviolet exposure), race, and NCCN risk category. Covariates were added to the model in an additive fashion, and covariates were kept in the model if $P<$ .10. All statistical tests were two-sided, with significance defined at .05. Additional regressions were conducted, stratified by NCCN category to assess trends within risk groups. Statistical analyses were conducted with Stata 12.1 (StataCorp, College Station, TX)

\section{RESULTS}

Overall, $45.8 \%$ (87) of the men in this cohort demonstrated adverse pathology at radical prostatectomy. The median age of men with adverse pathology was 65.0 years (interquartile range [IQR], 60.0 to 69.0 years), and the median age was 62.0 years (IQR, 60.0 to 69.0 years) for the men without adverse pathology $(P=.005)$. Similarly, men with adverse pathology demonstrated a statistically significant higher median BMI $\left(28.9 \mathrm{~kg} / \mathrm{m}^{2} v\right.$ $\left.27.7 \mathrm{~kg} / \mathrm{m}^{2} ; P=.04\right)$ and serum PSA $(6.8 \mathrm{ng} / \mathrm{mL} v 4.4 \mathrm{ng} / \mathrm{mL}$; $P<.001)$. In addition, men with adverse pathology at radical prostatectomy demonstrated lower median serum 25-OH D $(22.7 \mathrm{ng} / \mathrm{mL}$; IQR, 15.9 to 29.0$)$ compared with their counterparts $(27.0 \mathrm{ng} / \mathrm{mL}$; $\mathrm{IQR}, 20.0$ to $34.0 ; P=.007)$. Men with adverse pathology were also more likely to have a serum 25-OH D level less than $30 \mathrm{ng} / \mathrm{mL}$ $(80.5 \%$ v. $57.3 \% ; P=.001)$ and were more likely to self-identify as black $(P=.03)$. All other covariates did not demonstrate any significant differences on analysis (Table 1).

Pretreatment patient characteristics are listed in Table 2. Overall, $34.7 \%$ (66) of the men in this cohort met NCCN very lowor low-risk criteria; $68.4 \%$ (130) of the men presented with cT1c disease, $41.1 \%$ (78) had a Gleason score of $3+3 \mathrm{PCa}$, and $83.2 \%$ (158) had a PSA level of less than $10 \mathrm{ng} / \mathrm{mL}$. At the time of prostatectomy, $31.6 \%$ of the men demonstrated extraprostatic disease (pT3), with $10.0 \%$ having seminal vesicle invasion on final pathology (Table 3).

On multiple logistic regression, a serum 25-OH D level of less than $30 \mathrm{ng} / \mathrm{mL}$ (odds ratio [OR], 2.51; 95\% CI, 1.18 to $5.33 ; P=.02$ ) was associated with adverse pathology in a model controlling for age, serum PSA, and abnormal findings on digital rectal examination (Table 4). 25-OH D as a continuous variable did not have a significant association with adverse pathology for all men in the model. On stratified analysis, serum 25-OH D level (OR, 0.92; 95\% CI, 0.86-0.98; $P=.01$ ) and serum 25-OH D less than $30 \mathrm{ng} / \mathrm{mL}$ (OR, 3.62; 95\% CI, 1.15 to $11.46 ; P=.03$ ) were significantly associated with adverse pathology in men with NCCN intermediate PCa at diagnosis. There were no significant contributions to the models with race or season of blood draw added. Additionally, no significant associations were observed on multivariate analyses stratified by race/ethnicity with vitamin D. 


\begin{tabular}{|c|c|c|c|}
\hline Characteristic & Adverse Pathology $(n=87$ ) & Nonadverse Pathology $(n=103$ ) & $P$ \\
\hline \multicolumn{4}{|l|}{ Continuous, median (IQR) } \\
\hline Age, years & $65.0(60.0-69.0)$ & $62.0(58.0-66.0)$ & $.005^{*}$ \\
\hline Body mass index, $\mathrm{kg} / \mathrm{m}^{2} \dagger$ & $28.9(26.2-32.0)$ & $27.7(25.0-29.8)$ & $.04^{*}$ \\
\hline Serum PSA & $6.8(5.0-11.8)$ & $4.5(3.8-6.0)$ & $<.001^{*}$ \\
\hline 25-OH D serum level, $\mathrm{ng} / \mathrm{mL}$ & $22.7(15.9-29.0)$ & $27.0(20.0-34.0)$ & $.007^{*}$ \\
\hline \multicolumn{4}{|l|}{ Categorical, \% } \\
\hline First-degree family history of $\mathrm{PCa}(n=184)$ & 24.1 & 14.9 & .57 \\
\hline Abnormal findings on DRE $(n=189)$ & 44.2 & 18.5 & $<.001^{*}$ \\
\hline \multicolumn{4}{|l|}{ Race/ethnicity } \\
\hline Black $(n=45)$ & 32.2 & 16.5 & \\
\hline White $(n=128)$ & 56.3 & 76.7 & \\
\hline Other $(n=17)$ & 11.5 & 6.7 & $.03^{*}$ \\
\hline High school diploma or equivalent & 96.6 & 99.0 & .24 \\
\hline \multicolumn{4}{|l|}{ 25-OH D level, ng/mL } \\
\hline$<30$ & 80.5 & 57.3 & $.001 *$ \\
\hline$<20$ & 33.3 & 23.3 & 12 \\
\hline$<12$ & 16.1 & 7.8 & .07 \\
\hline Vitamin D supplement use & 16.1 & 22.3 & .28 \\
\hline Married & 75.9 & 76.7 & .89 \\
\hline Obesity $(n=188) \ddagger$ & 36.8 & 24.8 & .07 \\
\hline Tobacco use, current & 35.6 & 43.7 & .26 \\
\hline 5-ARI use $(n=187)$ & 2.4 & 3.9 & .54 \\
\hline \multicolumn{4}{|c|}{$\begin{array}{l}\text { NOTE. } P \text { values for continuous variables were derived from Wilcoxon rank-sum testing. } P \text { values for categorical variables were derived from } \chi^{2} \text { analysis. } \\
\text { Abbreviations: } 5-A R I, 5 \text { alpha reductase inhibitor; } 25-\mathrm{OH} \text { D, serum } 25 \text { hydroxyvitamin D; DRE, digital rectal examination; PCa, prostate cancer; PSA, prostate specific } \\
\text { antigen. } \\
\text { *Denotes statistic significance. } \\
\text { †BMl information was missing for one patient in this cohort. } \\
\text { †Obesity was defined as a BMl } \geq 30 \mathrm{ng} / \mathrm{mL} \text {. }\end{array}$} \\
\hline
\end{tabular}

\section{DISCUSSION}

Serum 25-OH D was consistently associated with adverse pathology on univariate and multivariate analysis in our cohort. Men with adverse pathology had a $15.9 \%$ lower serum $25-\mathrm{OH} \mathrm{D}$ level than did their counterparts. A 25-OH D level less than $30 \mathrm{ng} / \mathrm{mL}$ was associated with increased odds of adverse pathology, even after

\begin{tabular}{lr} 
Table 2. Pretreatment Characteristics of Patients in the Cohort $(\mathrm{N}=190)$ \\
\hline \multicolumn{1}{c}{ Characteristic } & No. (\%) \\
\hline Clinical TNM stage, N0/x, MO/x & \\
cT1c & $130(68.4)$ \\
cT2a & $32(16.8)$ \\
CT2b & $5(2.6)$ \\
cT2c & $23(12.1)$ \\
Gleason score at initial biopsy & \\
G3 + 3 & $78(41.1)$ \\
G3 + 4 & $52(27.3)$ \\
G4 + 3 & $28(14.7)$ \\
$\geq$ G4 + 4 & $32(16.8)$ \\
Serum PSA level (ng/mL) & \\
$\leq 10.0$ & $158(83.2)$ \\
$10.1-20.0$ & $24(12.6)$ \\
$>20.0$ & $8(4.2)$ \\
NCCN risk criteria & \\
Very low & $21(11.1)$ \\
Low & $45(23.7)$ \\
Intermediate & $85(44.7)$ \\
High & $39(20.5)$ \\
\hline
\end{tabular}

Abbreviations: NCCN, National Comprehensive Cancer Network; PSA, prostate specific antigen; TNM, tumor, node, metastasis staging system. controlling for well-known preoperative risk factors of adverse pathology, such as PSA level and disease palpable on digital rectal examination. ${ }^{6}$ This relationship seemed to be most pronounced among men with intermediate risk disease on stratified analysis; however, there was a nonsignificant association observed in men with low and low NCCN risk disease preoperatively.

There is sparse literature on the association of circulating serum 25-OH D and adverse pathology at the time of radical prostatectomy. To our knowledge, there is only one other study evaluating this relationship. Berg et $\mathrm{al}^{10}$ reviewed 100 consecutive men undergoing radical prostatectomy and did not find any correlation between Gleason score, pathologic staging, or positive margin status and vitamin $\mathrm{D}$ on multiple logistic regressions. The study, however, is limited by a small sample size. Given our sample size, we had $87 \%$ power to detect an OR greater than 2.5 for $25-\mathrm{OH} D$ levels less than $30 \mathrm{ng} / \mathrm{mL}$ and the presence of adverse pathology at radical prostatectomy, assuming a $35 \%$ rate of adverse pathology. ${ }^{12}$ The Berg et $\mathrm{al}^{10}$ study would have been underpowered to assess for this association, especially in adjusted analyses.

The correlation between vitamin D and PCa is being evaluated among men in a variety of basic science and translation research endeavors. Studies have demonstrated that PCa cells express the vitamin D receptor, which acts as a substrate in the activation and deactivation of many important cellular pathways. ${ }^{14-16}$ Specifically, vitamin $\mathrm{D}$ has been found to have an inhibitory effect on cellular proliferation, ${ }^{17,18}$ differentiation, ${ }^{17-20}$ and apoptosis. ${ }^{21-23}$

We have previously demonstrated an association of an increased overall risk of PCa in blacks and aggressive/high-risk disease both whites and blacks at the time of prostate biopsy among men with low vitamin D levels. ${ }^{3}$ Kristal et $\mathrm{al}^{1}$ demonstrated a U-shaped 


\begin{tabular}{|c|c|}
\hline Characteristic & No. (\%) \\
\hline \multicolumn{2}{|l|}{ Pathologic TNM stage $(N=190)$} \\
\hline pT2a & 22 (11.6) \\
\hline pT2b & $4(2.1)$ \\
\hline pT2c & $104(54.7)$ \\
\hline рТЗа & $41(21.6)$ \\
\hline рТ3b & $19(10.0)$ \\
\hline $\mathrm{pNx}$ & $3(1.6)$ \\
\hline pNO & $88(46.6)$ \\
\hline $\mathrm{pN} 1$ & $0(0.0)$ \\
\hline \multicolumn{2}{|c|}{ Pathologic Gleason score $(N=190)$} \\
\hline $\mathrm{G} 3+3$ & $48(25.4)$ \\
\hline $\mathrm{G} 3+4$ & 75 (39.7) \\
\hline $\mathrm{G} 4+3$ & $43(22.8)$ \\
\hline$\geq \mathrm{G} 4+4$ & $41(21.7)$ \\
\hline Unknown & $1(0.01)$ \\
\hline \multicolumn{2}{|c|}{ Adverse pathology by NCCN criteria $(n=87)$} \\
\hline Very low/low risk & $11(12.7)$ \\
\hline Intermediate risk & $43(49.4)$ \\
\hline High risk & $33(37.9)$ \\
\hline
\end{tabular}

relationship between vitamin D and PCa risk on the basis of prospective, multi-institutional data collected in the Selenium and Vitamin E Cancer Prevention Trial. Similarly, Schenck et $\mathrm{al}^{2}$ showed an increased risk of aggressive PCa among men with low vitamin D. Additionally, Steck et $\mathrm{al}^{24}$ showed that low 25-OH D levels were associated with aggressive PCa among black men in the North Carolina-Louisiana Prostate Cancer Project, and this relationship was modified negatively by low calcium intake. However, there are a couple of methodological differences-such as timing of blood draw, which was several months after PCa diagnosis in their cohort, and different risk strata definitions - that may explain disparities in some of the results from our respective cohorts. Nonetheless, our findings at the time of radical prostatectomy are consistent with the observations from these studies because vitamin D deficiency was associated with more aggressive disease findings at the time of radical prostatectomy in men with localized PCa. Unfortunately, Chicago, Illinois, is an ultraviolet-poor location, and elevated levels of vitamin $\mathrm{D}$ did not occur in this cohort.

The initial assessment of our Chicago cohort demonstrated a significant disparity regarding low vitamin D levels and black race compared with white men in both univariate and multivariate analyses. In fact, greater than $90 \%$ of black men in that study had vitamin D levels less than $30 \mathrm{ng} / \mathrm{mL} .{ }^{4}$ Interestingly, black men show a higher likelihood of progression and mortality on active surveillance for PCa. ${ }^{25}$ Data from the Johns Hopkins Hospital group suggest that failure on surveillance for black men may result from adverse pathologic features, as demonstrated in low-risk black men at the time of radical prostatectomy. ${ }^{9}$ Unfortunately, we could not verify any relationship between low serum vitamin $\mathrm{D}$ levels and adverse pathology on race-stratified univariate or multivariate analysis. Wes feel these analyses were significantly limited by a lack of power due to the relatively small size of each subgroup analyzed.

In addition, studies show a decrease in PSA velocity in men with advanced $\mathrm{PCa}$ receiving supplementation with calcitriol and closely related vitamin $\mathrm{D}$ analogs. ${ }^{27,28}$ The relationship between serum 25-OH D and PSA levels may be due to the effect of vitamin D on PCa cell proliferation and differentiation. Our findings showed that the largest association of low serum vitamin D was among men with intermediate NCCN risk disease at diagnosis. On the basis of the aforementioned data, this group may benefit most from normalization of their vitamin D levels with regard to their disease. Future studies should evaluate the effect of vitamin D levels and supplementation on $\mathrm{PCa}$ pathologic aggressiveness with regard to NCCN risk stratification, especially for men being considered for active surveillance, because vitamin D levels may be a useful biomarker in this population.

In a clinical setting, men with insufficient or deficient levels of 25-OH D at the time of PCa diagnosis may benefit from supplementation, with a goal of increasing serum $25-\mathrm{OH}$ D levels to a range of 30 to $55 \mathrm{ng} / \mathrm{mL}$. This could be achieved by assessing the serum vitamin D level at PCa diagnosis in all men with clinically localized disease prior to supplementation. An open-label trial of 4,000 IU of oral vitamin D3 in men undergoing active surveillance for favorable risk PCa demonstrated a 55\% decrease in the number of positive biopsies or Gleason grade at 1 year, with no adverse events among men in the study. ${ }^{26}$ However, we note that our recommendation needs to be substantiated with a large, randomized trial that would evaluate the impact of long-term vitamin D supplementation in men diagnosed with localized PCa.

The limitations of this study include its cross-sectional study design, which can lead to selection, observer, and analytical biases. The authors recognized these limitations as part of nested, retrospective analyses and adjusted for them by including all relevant

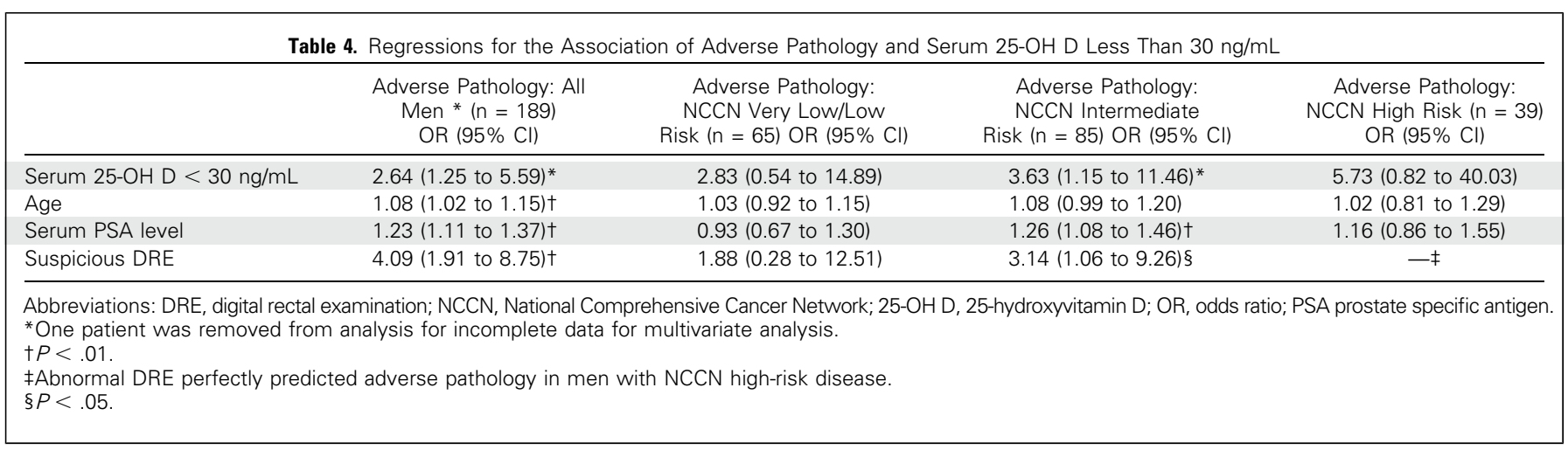


confounders. In addition, the sample size of the cohort diminished the power of many of the stratified analyses. A one-time measurement of serum 25-OH D has some limits as a proxy for overall vitamin $\mathrm{D}$ status in patients. In addition, adjustment for season of blood draw, which could confound associations with vitamin D levels, did not change our results. Lastly, men treated with radical prostatectomy at large, tertiary medical institutions may have limited generalizability to men treated in other settings. Nonetheless, our findings present new data that corroborate recent associations between PCa aggressiveness and low vitamin D levels demonstrated in a diverse population of men from a cohort in a large urban city.

In conclusion, insufficiency/deficiency of $25-\mathrm{OH} \mathrm{D}$ is associated with increased odds of adverse pathology in men with localized disease undergoing radical prostatectomy. Men with intermediate risk disease demonstrated the largest association between serum vitamin $\mathrm{D}$ levels and adverse pathologic findings at prostatectomy. Vitamin D could serve as an important biomarker of adverse pathology in men with $\mathrm{PCa}$, and the associations between PCa aggressiveness and vitamin D deserve continued exploration.

\section{AUTHORS' DISCLOSURES OF POTENTIAL CONFLICTS} OF INTEREST

Disclosures provided by the authors are available with this article at www.jco.org.

\section{AUTHOR CONTRIBUTIONS}

Conception and design: Yaw A. Nyame, Adam B. Murphy, Courtney M.P. Hollowell, Stephanie Kielb, Joshua J. Meeks, Peter H. Gann, Andre

Kajdacsy-Balla, William J. Catalona, Rick Kittles

Provision of study materials or patients: Andre Kajdacsy-Balla, William J. Catalona, Rick Kittles

Collection and assembly of data: Adam B. Murphy, Michael Dixon, Virgilia Macias, Andre Kajdacsy-Balla, Rick Kittles

Financial support: Rick Kittles

Administrative support: Rick Kittles

Data analysis and interpretation: Yaw A. Nyame, Adam B. Murphy, Diana K. Bowen, Gregory Jordan, Ken Batai, Virgilia Macias, Rick Kittles

Manuscript writing: All authors

Final approval of manuscript: All authors

\section{REFERENCES}

1. Kristal AR, Till C, Song $X$, et al: Plasma vitamin $D$ and prostate cancer risk: Results from the Selenium and Vitamin E Cancer Prevention Trial. Cancer Epidemiol Biomarkers Prev 23: 1494-1504, 2014

2. Schenk JM, Till CA, Tangen CM, et al: Serum 25-hydroxyvitamin $D$ concentrations and risk of prostate cancer: Results from the Prostate Cancer Prevention Trial. Cancer Epidemiol Biomarkers Prev 23:1484-1493, 2014

3. Murphy AB, Nyame Y, Martin IK, et al: Vitamin $D$ deficiency predicts prostate biopsy outcomes. Clin Cancer Res 20:2289-2299, 2014

4. Murphy AB, Kelley B, Nyame YA, et al: Predictors of serum vitamin $D$ levels in African American and European American men in Chicago. Am J Men Health 6:420-426, 2012

5. Epstein JI, Walsh PC, Carmichael M, et al: Pathologic and clinical findings to predict tumor extent of nonpalpable (stage T1c) prostate cancer. JAMA 271:368-374, 1994

6. D'Amico AV, Whittington R, Malkowicz SB, et al: Biochemical outcome after radical prostatectomy, external beam radiation therapy, or interstitial radiation therapy for clinically localized prostate cancer. JAMA 280:969-974, 1998

7. Epstein JI, Chan DW, Sokoll LJ, et al: Nonpalpable stage T1c prostate cancer: Prediction of insignificant disease using free/total prostate specific antigen levels and needle biopsy findings. J Urol 160: 2407-2411, 1998

8. Tosoian JJ, JohnBull E, Trock BJ, et al: Pathological outcomes in men with low risk and very low risk prostate cancer: Implications on the practice of active surveillance. J Urol 190:1218-1222, 2013

9. Sundi $D$, Ross $A E$, Humphreys $E B$, et al: African American men with very low-risk prostate cancer exhibit adverse oncologic outcomes after radical prostatectomy:
Should active surveillance still be an option for them? J Clin Oncol 31:2991-2997, 2013

10. Berg WT, Shapiro EY, Rothberg MB, et al: Baseline serum 25-hydroxyvitamin d levels in men undergoing radical prostatectomy: Is there an association with adverse pathologic features? Clin Genitourin Cancer 12:330-334, 2014

11. Kawachi MH, Bahnson RR, Barry $M$, et al: NCCN clinical practice guidelines in oncology: Prostate cancer early detection. J Natl Compr Canc Netw 8:240-262, 2010

12. Klein EA, Cooperberg MR, Magi-Galluzzi C, et al: A 17-gene assay to predict prostate cancer aggressiveness in the context of Gleason grade heterogeneity, tumor multifocality, and biopsy undersampling. Eur Urol 66:550-560, 2014

13. Etzioni RD, Howlader N, Shaw PA, et al: Longterm effects of finasteride on prostate specific antigen levels: Results from the prostate cancer prevention trial. J Urol 174:877-881, 2005

14. Ellfolk M, Norlin M, Gyllensten K, et al: Regulation of human vitamin $D(3) 25$-hydroxylases in dermal fibroblasts and prostate cancer $\mathrm{LNCaP}$ cells. Mol Pharmacol 75:1392-1399, 2009

15. Hendrickson WK, Flavin R, Kasperzyk JL, et al: Vitamin $D$ receptor protein expression in tumor tissue and prostate cancer progression. J Clin Oncol 29: 2378-2385, 2011

16. Miller GJ, Stapleton GE, Ferrara JA, et al: The human prostatic carcinoma cell line LNCaP expresses biologically active, specific receptors for 1 alpha,25-dihydroxyvitamin D3. Cancer Res 52:515-520, 1992

17. Blutt SE, Allegretto EA, Pike JW, et al: 1,25dihydroxyvitamin D3 and 9-cis-retinoic acid act synergistically to inhibit the growth of LNCaP prostate cells and cause accumulation of cells in G1. Endocrinology 138:1491-1497, 1997

18. Freedman LP: Transcriptional targets of the vitamin D3 receptor-mediating cell cycle arrest and differentiation. J Nutr 129:581S-586S, 1999 (2S, suppl)
19. Zhao XY, Ly LH, Peehl DM, et al: 1alpha,25dihydroxyvitamin D3 actions in LNCaP human prostate cancer cells are androgen-dependent. Endocrinology 138:3290-3298, 1997

20. Zhao $X Y$, Ly LH, Peehl DM, et al: Induction of androgen receptor by 1alpha,25-dihydroxyvitamin D3 and 9-cis retinoic acid in LNCaP human prostate cancer cells. Endocrinology 140:1205-1212, 1999

21. Blutt SE, McDonnell TJ, Polek TC, et al: Calcitriolinduced apoptosis in LNCaP cells is blocked by overexpression of Bcl-2. Endocrinology 141:10-17, 2000

22. Feldman $D$, Zhao $X Y$, Krishnan $A V$ : Vitamin $D$ and prostate cancer. Endocrinology 141:5-9, 2000

23. Sintov $A C$, Yarmolinsky $L$, Dahan $A$, et al: Pharmacological effects of vitamin $D$ and its analogs: Recent developments. Drug Discov Today 19:1769-1774, 2014

24. Steck $\mathrm{SE}$, Arab $\mathrm{L}$, Zhang $\mathrm{H}$, et al: Association between plasma 25-hydroxyvitamin $\mathrm{D}$, ancestry and aggressive prostate cancer among African Americans and European Americans in PCaP. PLoS One 10:e0125151, 2015

25. Mahal BA, Aizer AA, Ziehr DR, et al: Racial disparities in prostate cancer-specific mortality in men with low-risk prostate cancer. Clin Genitourin Cancer 12:e189-e195, 2014

26. Marshall DT, Savage SJ, Garrett-Mayer E, et al: Vitamin D3 supplementation at 4000 international units per day for one year results in a decrease of positive cores at repeat biopsy in subjects with low-risk prostate cancer under active surveillance. J Clin Endocrinol Metab 97:2315-2324, 2012

27. Gross C, Stamey T, Hancock S, et al: Treatment of early recurrent prostate cancer with 1,25-dihydroxyvitamin D3 (calcitriol). J Urol 159:2035-2039, 1998, discussion 2039-2040

28. Trump DL, Potter DM, Muindi $J$, et al: Phase II trial of high-dose, intermittent calcitriol (1,25 dihydroxyvitamin D3) and dexamethasone in androgen-independent prostate cancer. Cancer 106: 2136-2142, 2006 
Associations Between Serum Vitamin D and Adverse Pathology in Men Undergoing Radical Prostatectomy

The following represents disclosure information provided by authors of this manuscript. All relationships are considered compensated. Relationships are self-held unless noted. I = Immediate Family Member, Inst = My Institution. Relationships may not relate to the subject matter of this manuscript. For more information about ASCO's conflict of interest policy, please refer to www.asco.org/rwc or jco.ascopubs.org/site/ifc.

\section{Yaw A. Nyame}

No relationship to disclose

Adam B. Murphy

No relationship to disclose

Diana K. Bowen

No relationship to disclose

\section{Gregory Jordan}

No relationship to disclose

\section{Ken Batai}

No relationship to disclose

\section{Michael Dixon}

No relationship to disclose

Courtney M.P. Hollowell

No relationship to disclose

\section{Stephanie Kielb}

No relationship to disclose

Joshua J. Meeks

No relationship to disclose

Peter H. Gann

Research Funding: GlaxoSmithKline

Virgilia Macias

No relationship to disclose

Andre Kajdacsy-Balla

No relationship to disclose

William J. Catalona

Patents, Royalties, Other Intellectual Property: Co-inventor of urine assay for prostate specific antigen enzymatic activity

Rick Kittles

No relationship to disclose 